\title{
Update on COVID-19 vaccines and autoimmune-mediated rheumatic diseases
}

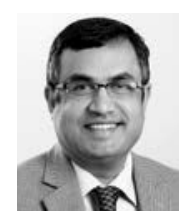

\author{
Lakshmanan Suresh ID \\ Department of Oral Diagnostic Sciences, University at Buffalo, NY, United States
}

Providing lasting long-term immunity through effective vaccines for current and emerging strains of coronavirus disease 2019 (COVID-19) providing lasting long-term immunity is fundamental to overcoming the pandemic. Recently approved COVID-19 vaccines have brought tremendous promise to help end this pandemic. As of July 20, 2021, the main vaccines that have been approved for use in North America and Europe are the mRNA vaccines BNT162b2 (Pfizer-BioNTech) and mRNA-1273 (Moderna), and the viral vector vaccines ChAdOx1 (AstraZeneca) and Ad26.COV2.S (Johnson \& Johnson) [1].

Autoimmune-mediated rheumatic diseases (ARDs) are a wide group of disorders that primarily affect the joints, bones, muscle, and connective tissue, affecting about $3-5 \%$ of the Western population [2, 3]. The optimal management of ARDs requires therapies that result in immunomodulation and immune suppression. This not only increases the risk of contracting COVID-19 infections but also can attenuate the vaccine-mediated antibody response. Multiple rheumatology organizations including the American College of Rheumatology, the European League Against Rheumatism (EULAR), the British Society of Rheumatology and the Canadian Rheumatology Association encourage and recommend COVID-19 vaccines, indicating that patients with ARDs should be encouraged to receive a COVID-19 vaccine, regardless of treatment regimen or underlying diagnosis $[4,5]$. The American College of Rheumatology (ACR) has recently published clinical guidance related to the timing of COVID-19 vaccination in relation to use of immunomodulatory therapies in ARD patients (Fig. 1).

They recommend not to delay COVID-19 vaccination for most immunomodulatory therapies except for rituximab (RTX). The panel further recommended vaccination to be scheduled such that the vaccine series would be initiated $\sim 4$ weeks prior to the next scheduled RTX dose. The ACR panel did not reach a consensus on ARD pa- tients receiving glucocorticoids at a prednisone-equivalent dose of $\geq 20 \mathrm{mg}$ per day [6].

Emerging data demonstrate reduced antibody responses in immunosuppressed individuals following mRNA vaccination [7, 8]. Among the immunosuppressed individuals there was heterogeneity of the humoral response to COVID-19 vaccines depending on the underlying condition. Organ transplant recipients receiving antimetabolite therapy and older recipients were less likely to develop an antibody response following the first dose of the BNT162b2 or the mRNA-1273 vaccines [7, 9]. Individuals with ARDs who received mRNA vaccines showed greater reduction in magnitude and quality of antibody responses compared to immunocompetent controls (Table I). Independent of dosage, glucocorticoid use showed a 10-fold reduction in antibody titers following SARS-CoV-2 vaccination. Moreover, use of prednisone in combination with other medications further attenuated antibody titers compared to other medications taken alone [8].

Currently, there is no consensus on the optimal antibody titers needed for protection and neutralization of the virus. There are gaps in knowledge on the correlates of vaccine-induced immune protection such as levels of neutralization, $\mathrm{B}$ cell memory responses and the role of the $T$ cell contribution in long-term immunity to COVID-19. Consequently, ACR guidelines do not advocate routine measurement of antibody levels after vaccination in ARD patients. However, there are emerging data on vaccine titers and efficacy. Recent studies of the BNT162b2 vaccines showed that the efficacy against the virus gradually decreased after attaining a peak of $96.2 \%$ after one month. The antibody titers gradually declined to $83.7 \%$, four months after dose 2 . There was an average decline in efficacy of approximately $6 \%$ every 2 months [10]. Ibarrondo et al. [11] reported that the antibodies developed from vaccination and natural mild infection showed similar characteristics in that their wane was slow but constant. There was an average decline of

Address for correspondence:

Lakshmanan Suresh, Department of Oral Diagnostic Sciences, University at Buffalo, 355 Squire Hall, South Campus, Buffalo, NY 14214, United States, e-mail: Isuresh@buffalo.edu

Submitted: 02.08.2021; Accepted: 05.08.2021 


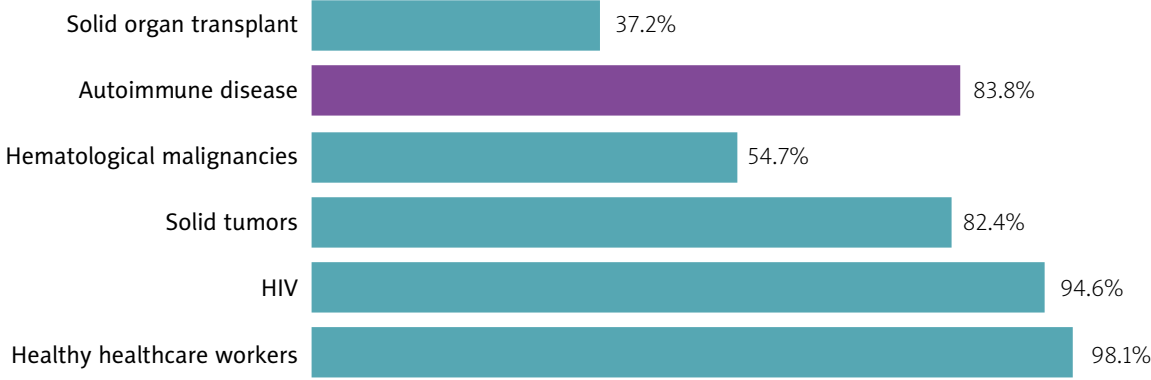

Extracted from Haidar G, Agha M, Lukanski A, et al. Immunogenicity of COVID-19 vaccination in immunocompromised patients: an observational, prospective cohort study interim analysis. medRxiv 2021, DOI: 10.1101/2021.06.28.21259576.

Fig. 1. Seropositivity rates in immunocompromised groups and healthy healthcare workers after COVID-19 vaccination.

Table I. ACR guidance related to the timing of COVID-19 vaccination in relation to use of immunomodulatory therapies in RMD patients*

\begin{tabular}{|c|c|c|}
\hline Medication(s) & $\begin{array}{l}\text { COVID-19 vaccine administration timing } \\
\text { considerations }\end{array}$ & Level of task force consensus \\
\hline $\begin{array}{l}\text { Hydroxychloroquine, sulfasalazine, } \\
\text { leflunomide, apremilast, IVIG }\end{array}$ & $\begin{array}{l}\text { Do not delay or adjust vaccine administration } \\
\text { timing }\end{array}$ & Strong \\
\hline $\begin{array}{l}\text { Methotrexate, mycophenolate mofetil, } \\
\text { azathioprine, cyclophosphamide } \\
\text { (IV or oral), TNFi, IL-6R, IL-1Ra, IL-17, IL-12/ } \\
\text { IL-23, IL-23, belimumab, JAK inhibitors, } \\
\text { abatacept (IV or SC), oral calcineurin } \\
\text { inhibitors, GCs (prednisone-equivalent } \\
\text { dose }<20 \mathrm{mg} / \text { day) })^{\dagger}\end{array}$ & $\begin{array}{c}\text { Do not delay or adjust vaccine administration } \\
\text { timing }\end{array}$ & Moderate \\
\hline Rituximab & $\begin{array}{l}\text { Assuming that a patient's COVID-19 risk is } \\
\text { low or able to be mitigated by preventive } \\
\text { health measures (e.g., self-isolation), schedule } \\
\text { vaccination so that the vaccine series is initiated } \\
\sim 4 \text { weeks prior to next scheduled rituximab cycle }\end{array}$ & Moderate \\
\hline
\end{tabular}

*COVID-19 - coronavirus disease 2019, RMD - rheumatic and musculoskeletal disease, IVIG - intravenous immunoglobulin, TNFi - tumor necrosis factor inhibitor, SC - subcutaneous. ' ${ }^{\dagger}$ Examples of cytokine and kinase inhibitors include the following: for interleukin-6 receptor (IL-6R), sarilumab and tocilizumab; for IL-1 receptor antagonist (IL-1Ra), anakinra and canakinumab; for IL-17, ixekizumab and secukinumab; for IL-12/IL-23, ustekinumab; for IL-23, guselkumab and risankizumab; for JAK inhibitors, baricitinib, tofacitinib and upadacitinib. Consensus was not reached for patients receiving glucocorticoids (CCs) at prednisone-equivalent doses of $\geq 20 \mathrm{mg} /$ day.

Extracted from PubMed Central as part of the COVID-19 public health emergency response. It can be used for unrestricted research re-use and analysis in any form or by any means with acknowledgement of the original source, for the duration of the public health emergency. Curtis JR, Johnson SR, Anthony DD, et al. American College of Rheumatology Guidance for COVID-19 vaccination in patients with rheumatic and musculoskeletal diseases - Version 2. Arthritis Rheumatol 2021; 73: 1093-1107, DOI: 10.1002/art.41877

antibody titers of approximately $90 \%$ in 3 months after infection or vaccination.

Similar findings were reported in another study of Pfizer-BioNTech mRNA vaccine in 118 subjects [12]. This study demonstrated a significant decline in antibody levels 6 weeks after the $2^{\text {nd }}$ dose. Older individuals showed a faster decline in their antibody levels and a weaker cellular immune response.

Our internal data (unpublished) indicate that antibody titers reach a peak 1 month after the $2^{\text {nd }}$ dose and there is a significant drop in antibody of over $70 \%$ 5 months after the $2^{\text {nd }}$ dose of the vaccine. Emerging and accumulating data point to waning vaccine-induced COVID immunity levels and a possible need for booster vaccination based on measurement of antibody levels, particularly in the immunocompromised, including ARD individuals. There is historical precedence for administering booster vaccines and for monitoring post-vaccine serology titers, especially in immunosuppressed and immunocompromised patients, and particularly in hepatitis $B$ patients. While routine monitoring of COVID-19 antibody responses is not yet advocated, there is increasing evidence that monitoring the antibody levels on a regular basis (six months to a year) may support optimal management of the patients.

The author declares no conflict of interest. 


\section{References}

1. Hazlewood GS, Pardo JP, Barnabe C, et al. Canadian Rheumatology Association recommendation for the use of COVID-19 vaccination for patients with autoimmune rheumatic diseases. J Rheumatol 2021, DOI: org/10.3899/jrheum.210288.

2. van der Heijde D, Daikh DI, Betteridge N, et al. Common language description of the term rheumatic and musculoskeletal diseases (RMDs) for use in communication with the lay public, healthcare providers and other stakeholders endorsed by the European League Against Rheumatism (EULAR) and the American College of Rheumatology (ACR). Ann Rheum Dis 2018; 77: 829-832, DOI: 10.1136/annrheumdis-2017-212565.

3. Hayter SM, Cook MC. Updated assessment of the prevalence, spectrum and case definition of autoimmune disease. Autoimmun Rev 2012; 11: 754-765, DOI: 10.1016/j.autrev.2012.02.001.

4. Kennedy NA, Goodhand JR, Bewshea C, et al. Anti-SARS-CoV-2 antibody responses are attenuated in patients with IBD treated with infliximab. Gut 2021; 70: 865-875, DOI: 10.1136/gutjnl-2021-324388.

5. Deepak P, Kim W, Paley MA, et al. Glucocorticoids and B cell depleting agents substantially impair immunogenicity of mRNA vaccines to SARS-CoV-2. medRxiv 2021, DOI: 10.1101/2021.04.05.21254656 [Preprint].

6. Curtis JR, Johnson SR, Anthony DD, et al. American College of Rheumatology Guidance for COVID-19 vaccination in patients with rheumatic and musculoskeletal diseases - Version 2. Arthritis Rheumatol 2021; 73: 1093-1107, DOI: 10.1002/ art.41877.

7. Boyarsky BJ, Werbel WA, Avery RK, et al. Immunogenicity of a single dose of SARS-CoV-2 messenger RNA vaccine in solid organ transplant recipients. JAMA 2021; 325: 1784-1786, DOI: 10.1001/jama.2021.4385.

8. Geisen UM, Berner DK, Tran F, et al. Immunogenicity and safety of anti-SARS-CoV-2 mRNA vaccines in patients with chronic inflammatory conditions and immunosuppressive therapy in a monocentric cohort. Ann Rheum Dis 2021, DOI: 10.1136/ annrheumdis-2021-220272 [Online ahead of print].

9. Haidar G, Agha M, Lukanski A, et al. Immunogenicity of COVID-19 vaccination in immunocompromised patients: an observational, prospective cohort study interim analysis. medRxiv 2021, DOI: 10.1101/2021.06.28.21259576.

10. Thomas SJ, Moreira Jr. ED, Kitchin N, et al. Six month safety and efficacy of BNT162b2 mRNA COVID-19 vaccine. medRxiv 2021, DOI: 10.1101/2021.07.28.21261159.

11. Ibarrondo JF, Hofmann C, Fulcher JA, et al. Primary, recall, and decay kinetics of SARS-CoV-2 vaccine antibody responses. ACS Nano 2021, DOI: 10.1021/acsnano.1c03972 [Online ahead of print].

12. Naaber P, Adamson A, Sepp E, et al. Antibody response after COVID-19 mRNA vaccination in relation to age, sex, and side effects. medRxiv 2021, DOI: 10.1101/2021.04.19.21255714. 